\title{
Pleural irrigation trial (PIT): a randomised controlled trial of pleural irrigation with normal saline versus standard care in patients with pleural infection
}

\author{
Clare E. Hooper ${ }^{1,2}$, Anthony J. Edey ${ }^{3}$, Anthony Wallis ${ }^{3}$, Amelia O. Clive ${ }^{1,2}$, \\ Anna Morley ${ }^{2}$, Paul White ${ }^{4}$, Andrew R.L. Medford ${ }^{2}$, John E. Harvey ${ }^{2}$, \\ Mike Darby ${ }^{3}$, Natalie Zahan-Evans ${ }^{2}$ and Nick A. Maskell ${ }^{1,2}$
}

Affiliations: ${ }^{1}$ Academic Respiratory Unit, School of Clinical Sciences, University of Bristol, Bristol, UK. ${ }^{2}$ Pleural Clinical Trials Unit, North Bristol Lung Centre, Southmead Hospital, Bristol, UK. ${ }^{3}$ Department of Radiology, Southmead Hospital, North Bristol NHS Trust, Bristol, UK. ${ }^{4}$ Statistical Department, University of West of England, Bristol, UK.

Correspondence: Nick Maskell, Reader in Respiratory Medicine, Academic Respiratory Unit, School of Clinical Sciences, University of Bristol, Bristol, BS10 5NB UK. E-mail: Nick.maskellabristol.ac.uk

ABSTRACT Pleural infection is increasing in incidence. Despite optimal medical management, up to $30 \%$ of patients will die or require surgery. Case reports suggest that irrigation of the pleural space with saline may be beneficial.

A randomised controlled pilot study in which saline pleural irrigation (three times per day for 3 days) plus best-practice management was compared with best-practice management alone was performed in patients with pleural infection requiring chest-tube drainage. The primary outcome was percentage change in computed tomography pleural fluid volume from day 0 to day 3 . Secondary outcomes included surgical referral rate, hospital stay and adverse events.

35 patients were randomised. Patients receiving saline irrigation had a significantly greater reduction in pleural collection volume on computed tomography compared to those receiving standard care (median (interquartile range) $32.3 \%(19.6-43.7 \%)$ reduction versus $15.3 \%(-5.5-28 \%)$ reduction) $(\mathrm{p}<0.04)$. Significantly fewer patients in the irrigation group were referred for surgery (OR 7.1, 95\% CI 1.23-41.0; $\mathrm{p}=0.03$ ). There was no difference in length of hospital stay, fall in C-reactive protein, white cell count or procalcitonin or adverse events between the treatment groups, and no serious complications were documented.

Saline irrigation improves pleural fluid drainage and reduces referrals for surgery in pleural infection. A large multicentre randomised controlled trial is now warranted to evaluate its effects further.

@ERSpublications

Does pleural irrigation improve pleural fluid drainage and resolution of sepsis in pleural infection? http://ow.ly/KPHeM

This article has supplementary material available from erj.ersjournals.com

Received: Aug 112014 | Accepted after revision: March 122015 | First published online: May 282015

Clinical trial: This study is registered at www.isrctn.com with identifier number 21646859.

Support statement: The trial was funded through an unrestricted education grant from North Bristol NHS Trust (grant number FSF08-2). Funding information for this article has been deposited with FundRef.

Conflict of interest: None declared.

Copyright OERS 2015 


\section{Introduction}

Pleural infection is a common and serious condition for which there is a lack of consensus regarding optimum management [1-7]. The annual incidence of pleural infection in the USA and UK combined is $\sim 65000$ [8]. Mortality from the disease is considerable: $\sim 20 \%$ in the 6 months following initial presentation $[7,9,10]$. Conservative management with intravenous antibiotics and chest tube drainage is the mainstay of medical therapy, but this fails in one-third of patients, necessitating surgical intervention [7]. However, the establishment of evidence-based management guidelines is hindered by wide variability in the clinical evolution of pleural infection and frequent baseline comorbidities of the affected population.

The natural history of para-pneumonic pleural infection progresses through increasing degrees of pleural fluid organisation at a rate and extent that varies between individuals [8]. Progressive loculation and organisation within the pleural space is a self-propagating process involving activation of pro-inflammatory cytokines and the coagulation cascade, making pleural fluid drainage increasingly difficult over time $[11,12]$. Disruption of the fibrinogenic pleural environment is a potentially important aspect of clinical management.

Pre-emptive surgical intervention is of unproven benefit and is not feasible for many patients. Evidence for intrapleural fibrinolytic therapies alone is lacking [7, 13-16]. However, positive results from the 2011 Multi-centre Intra-pleural Sepsis Trial (MIST 2) suggest that a combination of tissue plasminogen activator (t-PA) and DNase improves the chest radiographic appearance at day 7 [17], but this approach is expensive and requires further validation.

Intrapleural saline irrigation is a simple technique whereby physiological (0.9\%) saline is instilled into the pleural space via an in situ chest drain and allowed to drain out freely. A number of case series report promising results when it is used as an adjunct or alternative to surgery in the management of pleural infection [18-22]. This practice is a component of conservative management of pleural infection in several European centres, but is not yet supported by randomised clinical trial evidence.

We hypothesised that intrapleural saline irrigation would improve pleural fluid drainage and resolution of sepsis as part of the conservative management of patients presenting with pleural infection.

\section{Methods}

Study design

The trial was a pilot randomised controlled trial performed in a single centre in the UK.

\section{Subjects enrolled}

Trial entry was offered to all subjects fulfilling the entry criteria at Southmead Hospital (Bristol, UK). Subjects were screened in normal clinical practice. Screening logs recording reasons for non-trial entry were kept. For patients to be considered for the trial they needed to fulfil one of the following criteria. 1) Pleural fluid that was macroscopically purulent; 2) pleural fluid that was positive on culture for bacterial infection; 3) pleural fluid that was positive for bacteria upon Gram staining; or 4) pleural fluid that had a $\mathrm{pH}<7.2$ (measured in a blood gas analyser) in a patient with clinical evidence of infection.

Evidence of infection was assessed by the recruiting physician on the basis of fever, an elevated white-cell count and elevated serum inflammatory markers, e.g. C-reactive protein (CRP).

Study exclusion criteria were: 1) age $<18$ years; 2) inability to give informed written consent; 3) previous thoracic surgery or thrombolytic therapy for pleural infection; and 4) chest-tube placement $>72 \mathrm{~h}$ ago or size $>12$ French.

\section{Chest-tube drainage and antibiotic therapy}

All patients underwent chest-tube drainage with a 12-French Seldinger chest tube inserted under ultrasound guidance. All received i.v. antibiotics according to local hospital guidelines (see online supplementary material) and local microbiology advice.

Intravenous antibiotics were changed to oral consolidation therapy by the managing consultant in light of the clinical response. Antibiotic therapy was continued for between 2 and 6 weeks, as required.

\section{Allocation to trial groups}

After written informed consent, patients underwent a baseline thoracic computed tomography (CT) scan $\geqslant 12 \mathrm{~h}$ after chest tube insertion. Patients with a residual pleural collection despite chest-tube drainage were randomised in a 1:1 ratio using block randomisation and opaque sealed envelopes. The stratification criteria were: 1) $>25 \%$ or $<25 \%$ hemithorax volume occupied by pleural collection and 2) evidence of loculation on ultrasound. 


\section{Trial intervention}

Saline irrigation

250 - $\mathrm{mL}$ bags of $0.9 \%$ sodium chloride were administered through a giving set into the thoracic cavity via the chest tube and a three-way tap from a drip stand. Administration was by gravity (not an infusion pump) and no pressure was applied to increase the speed of the infusion, which was performed over $1 \mathrm{~h}$. The tube was then allowed to drain freely. This process occurred three times a day for a total of nine irrigations.

\section{Standard care}

In standard care, to maintain tube patency, $30-\mathrm{mL}$ normal saline flushes were given three times a day for 3 days. Thoracic suction (up to $-20 \mathrm{cmH}_{2} \mathrm{O}$ ) was applied to chest tubes in both groups between irrigations and flushes.

\section{CT imaging}

Multidetector volumetric CT data were acquired using an Aquilion CX scanner (Toshiba Medical Systems, Crawley, UK). This occurred at baseline (day $0, \geqslant 12 \mathrm{~h}$ after chest-tube insertion) and on day 3. Images of the whole thorax were obtained $45 \mathrm{~s}$ after injection of $75 \mathrm{~mL}$ iodinated intravenous contrast. Standard acquisition parameters were used: $40-350 \mathrm{~mA}$ (auto-modulated), $120 \mathrm{kVp}$, helical pitch factor 95 and $0.5 \mathrm{~s}$ rotation time. Scans were reconstructed at $1.5 \mathrm{~mm}$ and reviewed on a proprietary workstation using Vitrea software (Toshiba Medical Systems). Using the manual segmentation tool, the contour of each pleural collection was outlined, and the volume, in millilitres, automatically calculated by the software. Segmentation was performed separately by two experienced chest radiologists, blinded to clinical data or therapeutic intervention. Discrepancies (a difference of $>10 \%$ for volumes $>500 \mathrm{~mL}$, or $>50 \mathrm{~mL}$ for collections $<500 \mathrm{~mL}$ ) were reviewed and a consensus reached.

\section{Guidelines for the referral of patients for surgical intervention}

Patients were referred to the thoracic surgeons by the treating physician if there was a residual pleural collection with ongoing clinical sepsis, pyrexia or CRP $>100 \mathrm{mg} \cdot \mathrm{L}^{-1}$ despite optimal medical management. A decision to refer was made on day 3 , after the second CT thorax, or sooner if clinically indicated due to rapid deterioration.

\section{Trial outcomes}

Primary end-point

The primary outcome was the percentage change in pleural fluid volume on CT thorax from randomisation (day 0) to day 3 measured by two independent radiologists blinded to treatment allocation.

\section{Secondary end-points}

The secondary outcomes were 1) fall in inflammatory markers (absolute and percentage change) white cell count, CRP and procalcitonin from day 0 to day 3;2) time (hours) to becoming apyrexial in those with fever at baseline; 3) time (days) for CRP to fall below $100 \mathrm{mg} \cdot \mathrm{L}^{-1}$; 4) proportion of patients referred for thoracic surgery by 1 and 3 months; 5) duration of hospital stay from randomisation to discharge; 6) total volume of pleural fluid drained from randomisation to day 7; 7) all-cause mortality at 1 and 3 months; and 8) frequency of all, and serious, adverse events.

The consultant responsible recorded the reasons for referring patients for surgical treatment and the reasons for surgery. Each case was then reviewed by an independent reviewer blinded to patients' trial treatment prior to data analysis.

\section{Statistical analysis}

A written analysis plan was prepared and agreed before data unblinding and analysis. Subjects were excluded from the primary analysis if they did not receive any saline irrigation doses or control flushes, or if the repeat CT thorax was not performed due to clinical deterioration prompting urgent surgery.

All analyses were performed using GraphPad Prism (Version 5.0; GraphPad Software, La Jolla, CA, USA). Data are presented as mean \pm SD or median (interquartile range) as appropriate. Descriptive statistics were used to summarise patient characteristics. The t-test was used to examine differences between groups with parametric data and the Mann-Whitney U-test was used for nonparametric data. Fisher's exact test was used for categorical variables. Statistical significance was taken at the 5\% level.

\section{Power calculations}

As this was a pilot study no formal power calculation was possible, but published case reports and our own limited experience of using irrigation suggested a target recruitment of 35-40 patients with a $10 \%$ loss to follow-up rate would be appropriate for this pilot study. 


\section{Ethical approval and registration}

Ethical and regulatory approval for the study was obtained before recruitment commenced (UK research ethics committee reference 09/H0102/58). The trial was registered as a randomised study (ISRCTN number: 21646859)

\section{Results}

\section{Patients}

A flow chart showing the enrolment, assignment and follow-up of patients to the primary analysis is presented in figure 1. 47 participants were screened and 38 recruited to the trial between December 2009 and March 2012. Three patients were not randomised after initial CT thorax (no residual collection on CT scan; drain fell out; and patient transferred to the intensive care unit and was no longer able to consent). 35 participants received saline irrigation or standard care and 34 underwent repeat CT thorax on day 3. Thus the primary analysis included 34 participants (18 received saline irrigation and 16 received standard care).

The demographics and clinical characteristics of participants recorded at baseline are shown in table 1. The mean \pm SD age of participants was $60.5 \pm 17.97$ years and $26(74.2 \%)$ out of 35 were male. The characteristics of the treatment arms were similar and bacteriological and other clinical parameter data were similar to previous studies of pleural infection.

\section{Data quality}

The primary outcome measure (percentage change in volume $(\mathrm{mL})$ of pleural collection on CT imaging) was available in 34 out of 35 trial participants. One patient in the standard care arm required surgery due to clinical deterioration during day 2 , and therefore did not undergo a repeat CT thorax. There was no loss to follow-up, with 3-month post-randomisation data available on all participants.

\section{Primary end-point}

Saline irrigation resulted in a significantly larger reduction in CT pleural volume compared to standard care: irrigation group $32.3 \%$ (19.6-43.7\%) reduction versus standard care $15.3 \%(-5.5-28 \%)$ ( $\mathrm{p}<0.04)$ (fig. 2).

\section{Secondary end-points}

Length of hospital stay

Four patients (two in the irrigation arm and two in the control arm) required complex discharge planning after they were medically fit for discharge. Excluding these four patients, the median length of stay, measured from time of randomisation to discharge from hospital was 14 days (range 3-42 days) in the control group versus 8 days (4-36 days) in the irrigation arm $(\mathrm{p}=0.22)$.

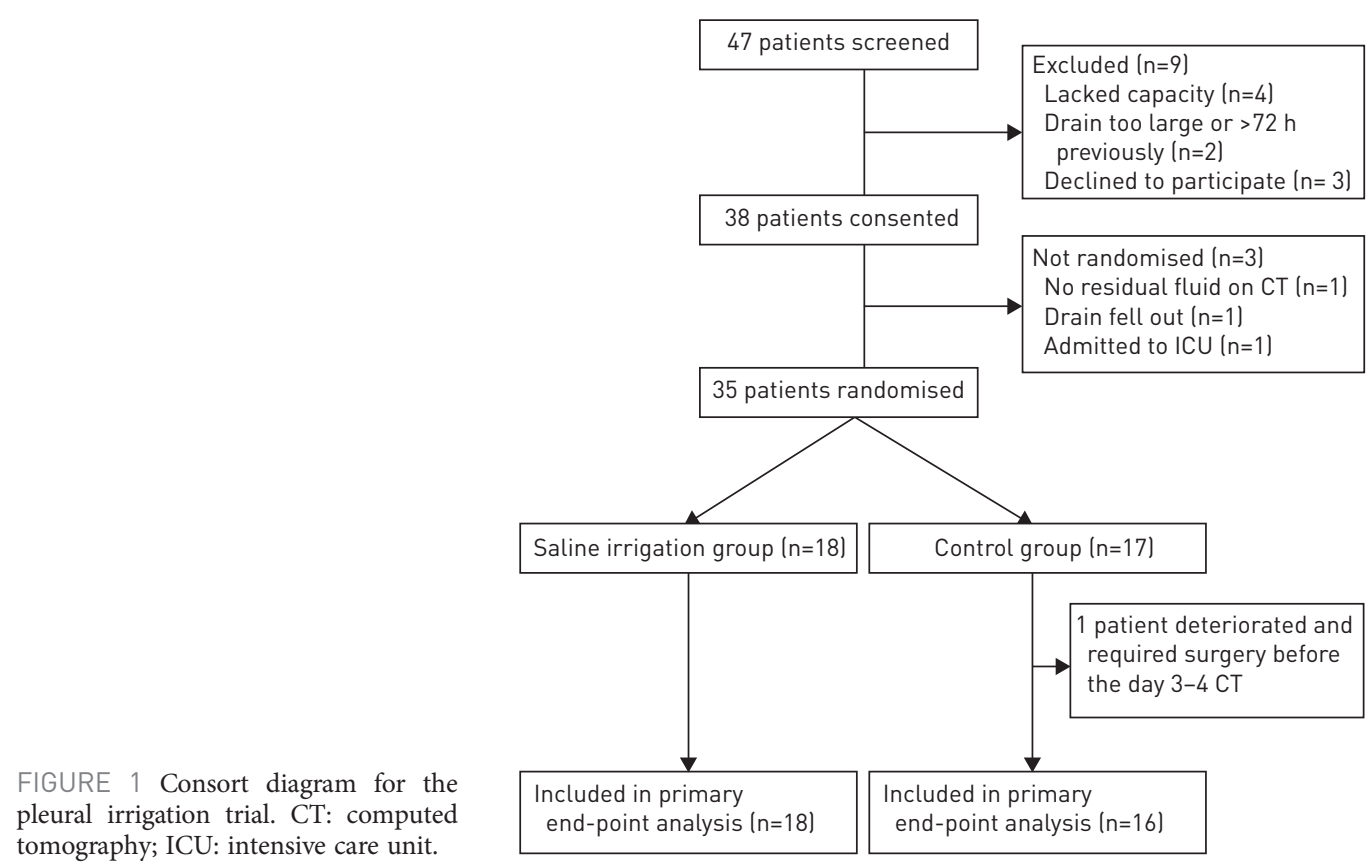


TABLE 1 Baseline demographic data of patients

\section{Saline irrigation}

\section{Patients $\mathrm{n}$}

Age years

Male:female ( $\%$ male)

Duration of symptoms prior to randomisation days

Pleural fluid protein $\mathrm{g} \cdot \mathrm{L}^{-1}$

Pleural fluid LDH IU. $\mathrm{L}^{-1}$

Pleural fluid $\mathrm{pH}$

C-reactive protein $\mathrm{mg} \cdot \mathrm{L}^{-1}$

Pleural fluid purulence n $(\%)$

Side of pleural collection (left:right)

Size of effusion on chest radiography

18
$56.7 \pm 17.6$
$13: 5(72)$
$14(5-35)$
$45.88 \pm 12.86$
$1605(139-3851)$
$6.90 \pm 0.16$
$98.65(36.8-144)$
$8(44)$
$7: 11$
$3(2-4)$

Standard care

Significant difference

$$
\begin{gathered}
64.5 \pm 18 \\
13: 4(76) \\
14(5-43) \\
40.63 \pm 9.46 \\
966(434-3162) \\
6.98 \pm 0.18 \\
120(69.25-240) \\
6(35) \\
6: 11 \\
3(2-4)
\end{gathered}
$$$$
17
$$

Data are presented as $\mathrm{n}$, mean \pm SD or median (interquartile range), unless otherwise stated. LDH: lactate dehydrogenase; NS: nonsignificant. ${ }^{\#}$ : measured using the method of LIGHT et al. [23].

Need for thoracic surgery

Compared to standard care, the irrigation group required less thoracic surgical intervention: eight out of 17 versus two out of 18 (OR 7.1, 95\% CI 1.23-41.0; $\mathrm{p}=0.03$ ) (fig. 3).

Review of the referrals for surgery was performed by an independent experienced physician blinded to the treatment allocation, and agreement occurred in all but one case. All referrals for surgery were made because of a persistent pleural collection with ongoing clinical evidence of sepsis (assessed by the managing physician in terms of fever, blood white count and CRP).

Total volume of pleural fluid drained

Compared to best standard care, the total volume of pleural fluid drained over the 6 days post-randomisation was increased in the irrigation group: $2075 \mathrm{~mL}(1050-2744 \mathrm{~mL})$ in the irrigation group versus $376 \mathrm{~mL}(287-632 \mathrm{~mL})$ in the standard care group $(\mathrm{p}<0.001)$. However, after subtracting the total amount of saline administered via the drain (saline irrigation and saline flushes) there was no statistical difference between the two groups ( $\mathrm{p}=0.255)$.

\section{Mortality}

Mortality rates were similar in both groups at 3 months (irrigation two out of 18; standard care: zero out of 17; $\mathrm{p}=0.49)$. Causes of death were not related to the pleural infection (small-bowel infarction $(n=1)$ and underlying malignancy $(\mathrm{n}=1))$.

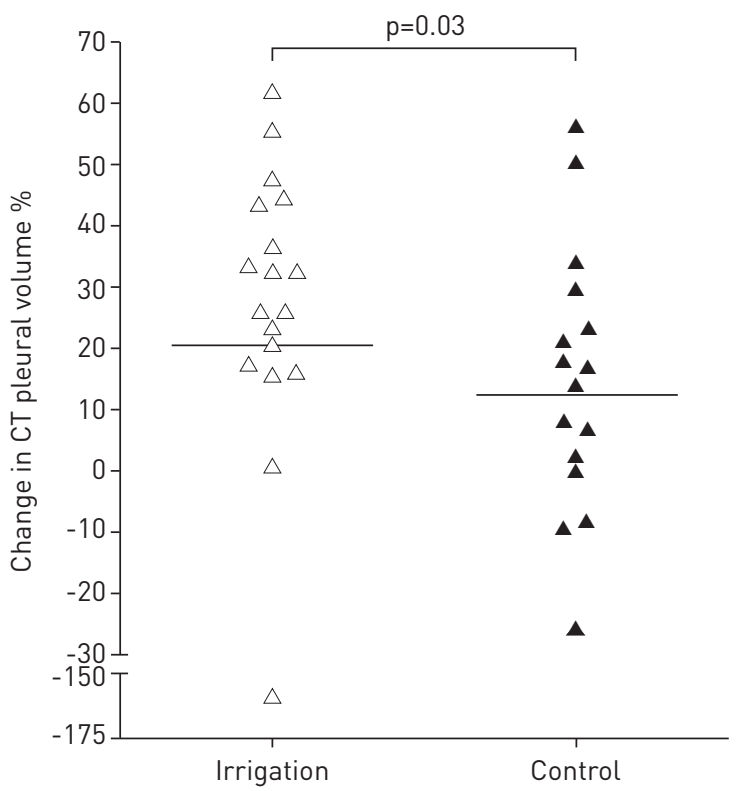

FIGURE 2 Percentage reduction in pleural volume (measured using computed tomography (CT)) at day 3 compared to day 0 . 
FIGURE 3 Numbers of patients requiring thoracic surgery.

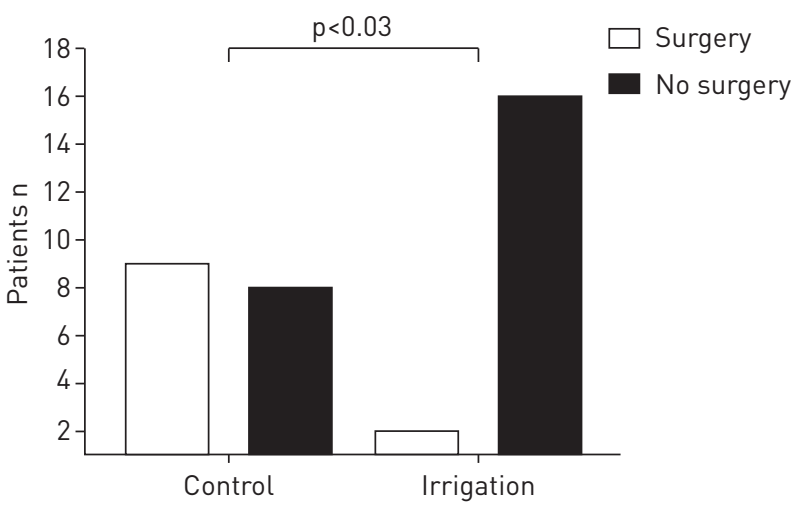

Changes in inflammatory markers and temperature

No differences were seen in the rate of falls in white cell count or CRP between the groups (day 0-day 3). There was also no difference in the time taken for the subjects to become apyrexial (5 days (range 06 days) in the irrigation arm versus 4.5 days (0-6 days) in the standard care arm).

\section{Changes in chest radiograph opacification}

The size of the effusion on baseline and 3-month follow-up chest radiographs were reviewed and scored by a radiologist blind to treatment allocation, using a previously described scoring system [23] (fig. 4).

\section{Lung function testing at 3-month follow-up}

Forced vital capacity (FVC) was recorded 3 months after randomisation and expressed as \% predicted. No differences were detected between the two groups: irrigation group $75.5 \pm 23.8 \%$ pred versus standard care group $78.5 \pm 13.8 \%$ pred $(\mathrm{p}=0.68)$.

\section{Compliance with saline irrigation and side-effects}

Saline irrigation was tolerated well with no significant serious side-effects noted. One patient had an undiagnosed bronchopleural fistula and could taste salty water in her mouth at the start of the first irrigation and therefore was not given further irrigations. One patient leaked saline from around the site of the drain insertion and another experienced localised chest discomfort during a single irrigation. Five out of 18 of the patients did not receive the full $250 \mathrm{~mL}$ normal saline during some of their irrigations, as it was administered under gravity and not forced in under pressure to minimise complications.

\section{Discussion}

These data suggest that in patients with pleural infection who have a residual pleural collection after initial chest tube drainage, treatment with intrapleural saline irrigation significantly reduces the size of the pleural collection when compared to standard medical management. There was a $32 \%$ reduction in pleural fluid volume on CT over 3 days in the irrigation group compared to a $15 \%$ reduction in patients treated with chest-tube drainage alone $(\mathrm{p}<0.04)$.

FIGURE 4 Chest radiograph scores at baseline and at 3-month follow-up.

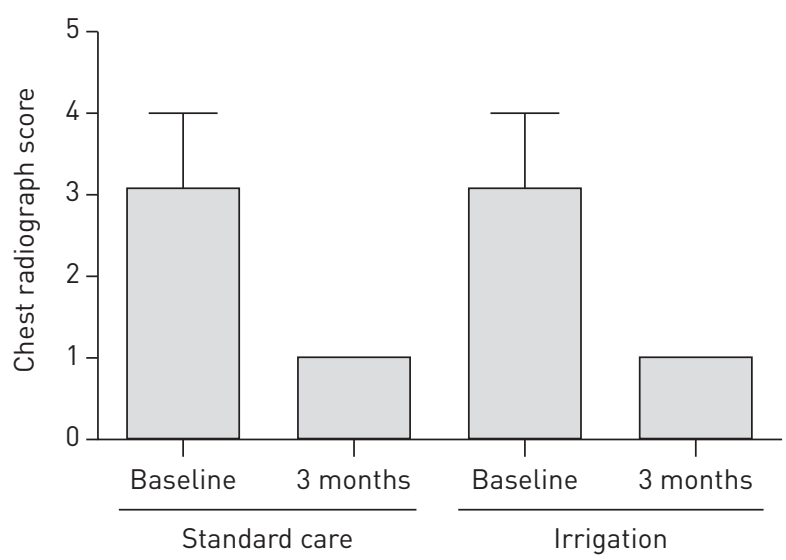


Although patient numbers in this pilot study are relatively small (34 included in the primary end-point analysis), the treatment allocation groups were shown to be well balanced both demographically and by their baseline chest radiograph opacification score, presence of loculation, purulence and markers of systemic sepsis.

The robust trial design adds to the likelihood of reproducibility of these results in larger future studies. We used manual segmentation of pleural collections on CT to measure our end-point, which is considered the radiological gold standard for volume measurements of pleural pathology [17, 24] and was performed independently by two radiologists blinded to treatment allocation.

The clinical relevance of improved pleural fluid drainage is reflected in significantly fewer patients requiring thoracic surgical intervention in the irrigation arm for poor resolution of systemic sepsis (two (11\%) out of 18 in the irrigation arm and eight (47\%) out of 17 in the control arm). Case review by an independent respiratory physician, blinded to treatment allocation, confirmed that decisions regarding surgical referral were clinically appropriate.

The association between improved radiographic resolution of the pleural collection and superior clinical outcomes in the irrigation group are similar to the results reported for the intrapleural t-PA plus DNase arm of the 2011 MIST 2 trial [17]. They showed an improvement of 58\% in chest radiograph opacification over 7 days with t-PA and DNase compared to our 32\% improvement in CT volume over 3 days. However, unlike the MIST 2 study we did not find a significant difference in the time taken for markers of systemic sepsis to settle. This might be due to our small sample size.

The optimum volume and frequency of irrigation is not known. The regime selected for this pilot study is consistent with that used in many European centres (Charles Marquette, University of Nice, Nice, France; personal communication), although up to $500 \mathrm{~mL}$ for each irrigation is used in some centres. Our study regime was not associated with serious or frequent side-effects and was easily deliverable by nursing staff in a standard respiratory ward setting. Further studies are required to look at the optimal delivery method and volume of saline irrigation.

There are at least two possible mechanisms to account for improved clinical outcomes: 1) removal and dilution of bacteria, cytokines, inflammatory cells and pro-fibrinogenic coagulation factors which propagate pleural fluid organisation [11, 12, 25-27]; and 2) reducing stasis and thus organisation of the intrapleural contents by the physical process of irrigation to increase fluid drainage. It could be hypothesised that both effects would be amplified if irrigation was initiated in early-stage pleural infection, thus further limiting pleural space fibrosis.

Another explanation for the reduced surgical referral rates in the irrigation group was the clear CT improvement on the day-3 scan. This scan was available to the treating physician when deciding whether to refer for surgery, and hence may have contributed to the decision-making process. It is reassuring to note that the appearance of chest radiographs and lung function (FVC) at 3 months were similar between the groups, indicating that the lack of surgical intervention in the irrigation arm did not affect long-term outcome in terms of respiratory compromise.

A limitation of the study is the potential for bias inherent in the trial design: the clinical team and patients were not blind to treatment allocation, which could affect clinical decision making. However, this had no impact on the primary end-point due to the blinding of the reporting radiologists. In addition, an independent observer blinded to treatment scrutinised the surgical referral decisions and demonstrated concordance with the unblinded clinicians.

Optimum management of patients with pleural infection will vary depending on individual comorbidities and access to healthcare resources. Saline irrigation is cheap, appears to be safe and well tolerated and may be particularly useful in patients for whom surgery is not an option.

This trial has shown that intrapleural saline irrigation improves clearance of the pleural space collection after initial chest-tube drainage when measured by CT, compared with standard care. No difference in hospital stay or fall of inflammatory markers was observed between the groups, but fewer participants required surgical intervention due to persistent pleural sepsis in the irrigation arm. There is now a compelling need for large randomised controlled studies using intrapleural saline irrigation, both alone and combination with other intrapleural therapies using the hard primary end-points of mortality and length of stay.

Acknowledgements

Thanks to Charles Marquette (University of Nice, Nice, France) for his advice about the irrigation regime.

\section{References}

1 Colice GL, Curtis A, Deslauriers J, et al. Medical and surgical treatment of parapneumonic effusions: an evidence-based guideline. Chest 2000; 118: 1158-1171. 
2 Berger HA, Morganroth ML. Immediate drainage is not required for all patients with complicated parapneumonic effusions. Chest 1990; 97: 731-735.

3 Davies RJ, Gleeson FV. The diagnosis and management of pleural empyema. Curr Opin Infect Dis 1998; 11: 163-168.

4 de Souza A, Offner PJ, Moore EE, et al. Optimal management of complicated empyema. Am J Surg 2000; 180: 507-511.

5 Waller DA, Rengarajan A, Nicholson FH, et al. Delayed referral reduces the success of video-assisted thoracoscopic debridement for post-pneumonic empyema. Respir Med 2001; 95: 836-840.

6 Davies HE, Davies RJ, Davies CW, et al. Management of pleural infection in adults: British Thoracic Society Pleural Disease Guideline 2010. Thorax 2010; 65: Suppl. 2, ii41-ii53.

7 Maskell NA, Davies CW, Nunn AJ, et al. U.K. controlled trial of intrapleural streptokinase for pleural infection. N Engl J Med 2005; 352: 865-874.

8 Light RW, Girard WM, Jenkinson SG, et al. Parapneumonic effusions. Am J Med 1980; 69: 507-512.

9 Ferguson AD, Prescott RJ, Selkon JB, et al. The clinical course and management of thoracic empyema. QJM 1996; 89: 285-289.

10 Davies CW, Kearney SE, Gleeson FV, et al. Predictors of outcome and long-term survival in patients with pleural infection. Am J Respir Crit Care Med 1999; 160: 1682-1687.

11 Alemán C, Alegre J, Monasterio J, et al. Association between inflammatory mediators and the fibrinolysis system in infectious pleural effusions. Clin Sci 2003; 105: 601-607.

12 Kroegel C, Antony VB. Immunobiology of pleural inflammation: potential implications for pathogenesis, diagnosis and therapy. Eur Respir J 1997; 10: 2411-2418.

13 Barletta JF. Streptokinase and urokinase for the treatment of pleural effusions and empyemas. Ann Pharmacother 1999; 33: 495-498.

14 Bouros D, Schiza S, Patsourakis G, et al. Intrapleural streptokinase versus urokinase in the treatment of complicated parapneumonic effusions: a prospective, double-blind study. Am J Respir Crit Care Med 1997; 155: 291-295.

15 Bouros D, Schiza S, Tzanakis N, et al. Intrapleural urokinase versus normal saline in the treatment of complicated parapneumonic effusions and empyema. A randomized, double-blind study. Am J Respir Crit Care Med 1999; 159: $37-42$.

16 Davies RJ, Traill ZC, Gleeson FV. Randomised controlled trial of intrapleural streptokinase in community acquired pleural infection. Thorax 1997; 52: 416-421.

17 Rahman NM, Maskell NA, West A, et al. Intrapleural use of tissue plasminogen activator and DNase in pleural infection. N Engl J Med 2011; 365: 518-526.

18 Mennander A, Laurikka J, Kuukasjärvi P, et al. Continuous pleural lavage may decrease postoperative morbidity in patients undergoing thoracotomy for stage 2 thoracic empyema. Eur J Cardiothorac Surg 2005; 27: 32-34.

19 Simmers TA, Jie C, Sie B. Minimally invasive treatment of thoracic empyema. Thorac Cardiovasc Surg 1999; 47: 77-81.

20 Karmy-Jones R, Sorenson V, Horst HM, et al. Rigid thorascopic debridement and continuous pleural irrigation in the management of empyema. Chest 1997; 111: 272-274.

21 Brutsche MH, Tassi GF, Györik S, et al. Treatment of sonographically stratified multiloculated thoracic empyema by medical thoracoscopy. Chest 2005; 128: 3303-3309.

22 Loddenkemper R, Kaiser D, Frank W. Treatment of parapneumonic pleural effusion and empyema: conservative view. In: Verleden GM, Van Raemdonck D, Lerut T, et al., eds. Surgery for Non-Neoplastic Disorders of the Chest: a Clinical Update (ERS Monograph). Sheffield, European Respiratory Society, 2004; pp. 199-207.

23 Light RW, Rogers JT, Cheng D, et al. Large pleural effusions occurring after coronary artery bypass grafting. Ann Intern Med 1999; 130: 891-896.

24 Zhao B, Schwartz L, Flores R, et al. Automated segmentation of mesothelioma volume on CT scan. Proc Int Soc Optics Phototonics 2005; 5747: 866-871.

25 Sasse SA, Causing LA, Mulligan ME, et al. Serial pleural fluid analysis in a new experimental model of empyema. Chest 1996; 109: 1043-1048.

26 Idell S, Girard W, Koenig KB, et al. Abnormalities of pathways of fibrin turnover in the human pleural space. Am Rev Respir Dis 1991; 144: 187-194.

27 Sahn SA, Reller LB, Taryle DA, et al. The contribution of leukocytes and bacteria to the low $\mathrm{pH}$ of empyema fluid. Am Rev Respir Dis 1983; 128: 811-815. 